\title{
EINE LOGISCHE REKONSTRUKTION DER PLATONISCHEN PRÄDIKATIONSTHEORIE
}

\author{
Uwe MEIXNER \\ Universität Regensburg
}

1.

Unter einer Theorie der (einstelligen) Prädikation (1. Stufe) verstehe ich einen Explikationsvorschlag für den Ausdruck ,(der Begriff) $f$ trifft auf (den Gegenstand) $x$ zu“. Die erste Prädikationstheorie in der abendländischen Philosophie wurde von Platon entwickelt; seine Ideenlehre mit der darin enthaltenen Lehre von der metexis (der Teilhabe) läßt sich nämlich als eine Prädikationstheorie deuten.

2.

Platons Prädikationstheorie weicht in einigen Punkten von unseren modernen Auffassungen stark ab:

(a) Begriffe ${ }^{1}$ sind bei Platon nicht kategorial von Gegenständen verschieden; vielmehr bilden die Begriffe (die Ideen oder Formen) eine Unterklasse der Gegenstände; sie sind Gegenstände, allerdings keine gewöhnlichen; sie sind nicht sinnlich wahrnehmbar, sie sind unwandelbar und sie sind vollkommener als die gewöhnlichen Gegenstände. (Vgl. hierzu das 1. Zitat im Appendix.)

(b) Mit der Angleichung der Begriffe an Gegenstände geht einher, daß Begriffe auf sich selbst zutreffen können. Bei Platon treffen auch tatsächlich alle Begriffe, an die er zunächst denkt (Schönheit, Gerechtigkeit etc.), auf sich selbst zu; die Schönheit z.B. ist schön (siehe das 1.Zitat im Appendix). Wenn dagegen Begriffe kategorial von Gegenständen verschieden sind, dann kann kein Begriff auf sich selbst zutreffen, da die zweite Stelle der Prädikationsrelation stets von einem Gegenstand ausgefüllt werden muß.

1. Der Gebrauch des Wortes „Begriff“ sei hier völlig neutral; insbesondere ist es von fregeschen Assoziationen freizuhalten. 
(c) Die Prädikationsrelation läßt Grade zu. Ein Begriff kann auf den Gegenstand a in höherem Grade zutreffen als auf den Gegenstand b. Dazu, daß man schlechthin vom Zutreffen eines Begriffes auf einen Gegenstand sprechen kann, ist es hinreichend und notwendig, daß der Begriff in einem bestimmten Mindestgrad auf den Gegenstand zutrifft. Es gibt auch einen maximalen Grad des Zutreffens; die Schönheit z.B. ist im höchsten Grade schön. Begriffe (wenigstens alle die, an die Platon zunächst denkt) treffen in maximalem Grade auf sich selbst zu; auf gewöhnliche Gegenstände treffen sie dagegen nur in geringerem als maximalem Grade zu. (Vgl. hierzu das 2. Zitat im Appendix.)

(d) Für alle Begriffe $f$ und Gegenstände $x$ gilt: wenn $f$ im maximalen Grade auf $x$ zutrifft, so ist $x$ mit $f$ identisch; umgekehrt: ist $x$ mit $f$ identisch, dann trifft $f$ im maximalen Grade auf $x$ zu. ${ }^{2}$ Damit ergibt sich, daß ein Begriff auf sich selbst und nur auf sich selbst im maximalen Grade zutrifft.

3.

Den angesprochenen Verschiedenheiten der platonischen Prädikatstheorie von der unsrigen liegt ein einheitliches Prädikationsparadigma zugrunde, das völlig anders ist als unser Paradigma der Prädikation als Erfüllungs- und Sättigungsrelation. Platon konzipiert die Prädikation nicht als Erfüllungsrelation, sondern als Ähnlichkeitsrelation: ${ }^{3}$

(Sokrates spricht) Sondern, o Parmenides, eigentlich scheint es mir sich so zu verhalten, daß nämlich diese Begriffe gleichsam als Urbilder dastehen in der Natur, die andern Dinge aber diesen gleichen und Nachbilder sind, und daß die Aufnahme der Begriffe in die andern Dinge nichts anderes ist, als da $B$ diese ihnen nachgebildet werden. (Parmenides, 132d; in der Übersetzung von F. Schleiermacher)

2. Im Sinne Platons gesprochen: Das Schöne an sich ist am schönsten, also ist es die Schönheit selbst; weil das Schöne an sich die Schönheit ist, ist es am schönsten.

3. Dies scheint mir, insgesamt betrachtet, auch die Deutung der Teilhabe zu sein, die Platon selbst schließlich favorisierte. Ich kann mich hier aber natürlich nicht auf die Komplikationen der Platon-Interpretation einlassen; das wăre ein zu weites Feld. 
4.

Ich möchte nun eine logische Rekonstruktion ${ }^{4}$ der platonischen Prädikationstheorie im Rahmen der Prädikatenlogik 1. Stufe mit Identität angeben. D.h. ich verwende nur einen Typus von Variablen: kleine Buchstaben aus dem hinteren Teil des Alphabets: $x, y, r, r$ ', x', $y^{\prime}$..., die intersubstituierbar mit Objektkonstanten sind. Statt logischer Symbole benütze ich zum großen Teil, der besseren Lesbarkeit halber, an der Umgangssprache orientierte Kürzel.

\section{(a) Nichtlogische Grundausdrücke}

Zahlennamen (in Standardnotation);

$r^{*}$, lies ,der Mindestgrad“;

$\mathrm{G}(\mathrm{x})$, lies ,x ist ein Gegenstand“;

$\mathrm{B}(\mathrm{x})$, lies „, $\mathrm{x}$ ist ein Begriff (ein Standardgegenstand, eine Form, eine Idee)";

$R(x)$, lies , $x$ ist eine reelle Zahl“;

$\mathrm{S}(\mathrm{x}, \mathrm{y}, \mathrm{r})$, lies , $\mathrm{x}$ ist y mindestens im Grade $\mathrm{r}$ ähnlich“;

$\mathrm{x}<\mathrm{y}$, lies , $\mathrm{x}$ ist eine kleinere reelle Zahl als $\mathrm{y}^{\text {“; }}$

$(\mathrm{x} \leq \mathrm{y}:=\mathrm{x}<\mathrm{y}$ oder (o.) $[\mathrm{R}(\mathrm{x})$ und (u.) $\mathrm{R}(\mathrm{y}) \mathrm{u} . \mathrm{x}=\mathrm{y}]$ ).

\section{(b) Axiome}

$R(\rho)$, für jeden Zahlennamen $\rho$;

Axiome, die < in üblicher Weise im Zusammenhang mit $\mathbf{R}$ charakterisieren;

A1 $\forall x(B(x)$ impliziert (imp.) $\mathrm{G}(\mathrm{x}))-$,Jeder Begriff ist ein Gegenstand“;

4. Ziel der logischen Rekonstruktion einer älteren philosophischen Theorie (einer Theorie, die einer solchen Behandlung fähig ist) ist, sie unserem Verständnis näher zu bringen, und zwar in einer exakteren und kohärenteren Weise, als es durch andere Interpretationsmethoden möglich ist. Interpretieren heißt immer, in anderen Worten als denen des Autors klar zu sagen, was der Autor bei wohlwollender Deutung meinte, und eine Interpretation ist nicht um so besser, je mehr sie in ihrem eigenen Wortlaut am Wortlaut des Textes bleibt. Darum ist es kein Einwand gegen die Methode der logischen Rekonstruktion, da sie von Konzepten (etwa dem der reellen Zahl) Gebrauch macht, die Platon z.B. nicht explizit anwendete. Angedeutet finden sich einige der Gedanken zu Platons Ideenlehre, die ich hier verfolgen werde, schon in einem Aufsatz von P.T. Geach aus dem Jahre 1956: „The Third Man Again“, Philosophical Review 65, 72-82. 
A2 $\forall x(G(x)$ imp. non $R(x))-$,Kein Gegenstand ist eine reelle Zahl“;

A3 $0.5<r^{*}<1-$, ,Der Mindestgrad ist größer als 0.5 , aber kleiner als 1“;

A4 $\forall \mathrm{x} \forall \mathrm{y} \forall \mathrm{r}(\mathrm{S}(\mathrm{x}, \mathrm{y}, \mathrm{r})$ imp. $\mathrm{G}(\mathrm{x})$ u. $\mathrm{G}(\mathrm{y})$ u. $0 \leq \mathrm{r} \leq 1)-$,Wenn $\mathrm{x}$ y mindestens im Grade $r$ ähnlich ist, dann ist $x$ ein Gegenstand und $\mathrm{y}$ ein Gegenstand und $\mathrm{r}$ ist mindestens 0 , aber höchstens $1^{\text {“; }}$

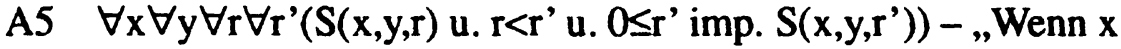
$y$ mindestens im Grade $r$ ähnlich ist und $r$ ' kleiner als $r$, aber mindestens 0 ist, dann ist $x$ y mindestens im Grade r' ähnlich“;

A6 $\forall \mathbf{x} \forall \mathbf{y} \forall \mathrm{r}(\mathbf{S}(\mathrm{x}, \mathrm{y}, \mathrm{r})$ imp. $\mathrm{S}(\mathrm{y}, \mathrm{x}, \mathrm{r}))-$,Wenn $\mathrm{x}$ y mindestens im Grade $r$ ähnlich ist, dann ist $y x$ mindestens im Grade $r$ ähnlich";

A7 $\forall x \forall y(G(x)$ u. $G(y)$ imp. ( $x=y$ äqu. $S(x, y, 1)))-$ „Wenn $x$ und $y$ Gegenstände sind, dann ist $x$ mit $y$ genau dann identisch, wenn $x$ y mindestens im Grade 1 ähnlich ist“;

A8 $\forall \mathrm{x} \forall \mathrm{y}\left(\mathrm{G}(\mathrm{x})\right.$ u. $\mathrm{G}(\mathrm{y})$ imp. $\operatorname{Vr}\left(\mathrm{S}(\mathrm{x}, \mathrm{y}, \mathrm{r})\right.$ u. non $\mathrm{Vr}^{\prime}\left(\mathrm{r}<\mathrm{r}^{\prime} \mathrm{u}\right.$. $\left.\left.\left.\mathrm{S}\left(\mathrm{x}, \mathrm{y}, \mathrm{r}^{\prime}\right)\right)\right)\right)$ - , Wenn $\mathrm{x}$ und y Gegenstände sind, dann gibt es einen Grad, in genau dem $x$ y ähnlich ist““.

(c) Definitionen

$x^{r}(y):=S(y, x, r)$ u. $B(x)$ u. $r^{*} \leq r$

$(, x$ trifft auf $y$ mindestens im Grade $r z u:=y$ ist dem Begriff $x$ mindestens im Grade r, der mindestens so groß wie der Mindestgrad ist, ähnlich")

$x(y):=x^{r^{*}}(y)$

(, $x$ trifft auf y $z u:=x$ trifft auf y mindestens im Mindestgrad $\left.z u^{\prime c}\right)$ $x^{+}(y):=x^{1}(y)$

(,x trifft auf y im höchsten Grade $z u:=x$ trifft auf y mindestens im Grade $1 \mathrm{zu}^{\text {“") }}$

$x^{-7}(y):=x^{r}(y)$ u. non $\exists r^{\prime}\left(r<r^{\prime}\right.$ u. $\left.x^{r^{\prime}}(y)\right)$

(,x trifft auf y [genau] im Grade $r$ zu :=x trifft auf y mindestens im Grade $r \mathrm{zu}$, und es gibt keinen höheren Grad, in dem $x$ auf $y$ mindestens zutrifft")

$x^{>}(y / z):=\exists r \exists r^{\prime}\left(x^{=r}(y)\right.$ u. $x^{=r^{\prime}}(z)$ u. $\left.r^{\prime}<r\right)$

(,x trifft auf $y$ in höherem Grade zu als auf $z:=$ es gibt Grade $r$ und $r^{\prime}$, in denen $x$ auf $y$, respektive $z$ zutrifft, und $r$ ' ist kleiner als $r^{\prime \prime}$ ) $N(x):=G(x)$ u. non $B(x)$ 
(,x ist ein gewöhnlicher Gegenstand := $\mathrm{x}$ ist ein Gegenstand, aber kein Begriff")

\section{(d) Theoreme}

T1 $\forall x(G(x)$ imp. $S(x, x, 1))-$,Jeder Gegenstand ist sich selbst mindestens im Grade 1 ähnlich", aus A7;

T2 $\forall x(B(x)$ äqu. $x(x))$ - „Die Begriffe sind genau die Entitäten, die auf sich selbst zutreffen"; aus A1, T1, A3, A5 und den Definitionen;

T3 $\forall x\left(B(x)\right.$ äqu. $\left.x^{+}(x)\right)-$ „Die Begriffe sind genau die Entitäten, die im höchsten Grade auf sich selbst zutreffen"; dem Beweis von T2 kann man den Beweis von T3 entnehmen;

T4 $\forall x \forall y\left(x^{+} y\right)$ äqu. $B(x)$ u. $\left.y=x\right)-,, x$ trifft im höchsten Grade auf $y$ genau dann zu, wenn $x$ ein Begriff und $y$ mit $x$ identisch ist"; aus den Definitionen, A4 und A7; A1, T1, A3 und den Definitionen;

T5 $\forall \mathrm{x}(\mathrm{N}(\mathrm{x})$ äqu. $\mathrm{G}(\mathrm{x})$ u. non $\mathrm{x}(\mathrm{x}))-$,Die gewöhnlichen Gegenstände sind die Gegenstände, die nicht auf sich selbst zutreffen"; aus T2 und den Definitionen;

T6 $\forall x \forall y\left(x(y) \operatorname{imp} \exists r\left(x^{=r}(y)\right)\right)-$, ,Wenn $x$ auf y zutrifft, dann gibt es einen Grad, in dem $x$ auf y zutrifft"; aus den Definitionen, $A 4, A 8$;

T7 $\forall x \forall y\left(B(x)\right.$ u. $N(y)$ u. $x(y)$ imp. $\left.x^{>}(x / y)\right)-$, ,Jeder Begriff, der auf einen gewöhnlichen Gegenstand zutrifft, trifft in höherem Grade als auf diesen auf sich selbst zu“; Beweis: angenommen $\mathrm{B}(\mathrm{x}), \mathrm{N}(\mathrm{y}), \mathrm{x}(\mathrm{y})$; wegen $\mathrm{B}(\mathrm{x})$ folgt nach $\mathrm{T}^{3} \mathrm{x}^{+}(\mathrm{x})$, also $\mathrm{x}^{1}(\mathrm{x})$; nun non $\exists \mathrm{r}^{\prime}\left(1<\mathrm{r}^{\prime} \mathrm{u}\right.$. $\left.\mathrm{x}^{\mathrm{r}^{\prime}}(\mathrm{x})\right)$, denn aus $\mathrm{x}^{\mathrm{r}^{\prime}}(\mathrm{x})$ folgt $S\left(x, x, r^{\prime}\right)$, also mit $A 4 r^{\prime} \leq 1$, also non $1<r^{\prime} ;$ also $x^{=1}(x)$; aus $x(y)$ folgt nach $T 6 \exists r^{\prime}\left(x^{=r^{\prime}}(y)\right) ; r^{\prime}<1$, denn sonst: aus $x^{=x^{\prime}}(y)$ nach den Definitionen $x^{r^{\prime}}(y)$, also $S\left(y, x, r^{\prime}\right)$, also mit $1 \leq r^{\prime}$ und $A 5$ $S(y, x, 1)$, also mit $A 7(d a G(y), G(x)) y=x$ - was unmöglich ist, da $N(y)$; demnach $x^{=1}(x)$ u. $\exists r^{\prime}\left(x^{-r^{\prime}}(y)\right.$ u. $\left.r^{\prime}<1\right)$, also $\exists r \exists r^{\prime}\left(x^{=}(x)\right.$ u. $x^{\prime \prime}(y)$ u. $\left.r^{\prime}<r\right)$ also $x^{>}(x / y)$.

\section{5.}

Die platonische Wendung ,x hat Teil an $\mathrm{f}^{\prime \prime}$ (formal , $\mathrm{x}$ par $\mathrm{f}$ ") wird man wiedergeben durch , $N(x)$ u. $f(x)$ “. (Man beachte, da $B$ danach kein Begriff an sich selbst Teil hat, denn kein Begriff ist ein 
gewöhnlicher Gegenstand; nur gewöhnliche Gegenstände haben Teil an etwas; dies entspricht platonischer Lehre, siehe dazu unten Abschnitt 8.) Jedoch die platonische Behauptung, „Die Schönheit ist schön" können wir bislang nicht wiedergeben (und also nicht beweisen), sondern nur ihre Entsprechung „Die Schönheit trifft auf die Schönheit zu“" (wenn wir $\lambda S$ - lies „die Schönheit“ - mit B( $\lambda S$ ) einführen). Wir erweitern nun unsere formale Sprache um Beinamen (in der Umgangssprache entsprechen den Beinamen die Adjektive)

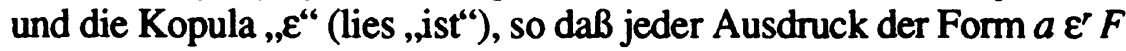
(,a ist mindestens im Grade $\mathrm{r} \mathrm{F}^{\prime}$ ), bzw. der Form $a \varepsilon^{=r} F$ (,a ist [genau] im Grade $r$ F") ein Satz ist, falls a ein Name, $r$ ein Name und $F$ ein Beiname ist. (Beinamen sind keine Namen, sondern stehen bei Namen; sie sind deshalb nicht quantifizierbar.) Außerdem erweitern wir sie um den Operator $\lambda$ (lies „die ...-heit“), der aus jedem Beinamen den zugehörigen Begriffsnamen (einen Gegenstandsnamen) bildet (,gerecht" - „Gerechtigkeit“, bzw. „das Gerechte“; „schön“ - „die Schönheit“, bzw. „das Schöne“; „gut“ - „die Gutheit", bzw. „das Gute“; etc.). - Dann postulieren wir die folgenden zwei Axiomenschemata:

A9 $B(\lambda F)$, für alle Beinamen $F$

A10 $\forall x \forall r\left(x \varepsilon^{[r]} F\right.$ äqu. $\left.\lambda F^{[r]}(x)\right)$, für alle Beinamen $F$; für ,[r] " ist einzusetzen , $r "$ oder aber,$=r$ ".

Und wir definieren

$\mathrm{x} \varepsilon \mathrm{F}:=\mathrm{x} \varepsilon^{\boldsymbol{r}^{*}} \mathrm{~F}$

(, $\mathrm{x}$ ist $\mathrm{F}:=\mathrm{x}$ ist mindestens im Mindestgrad $\mathrm{F}^{\mathrm{c}}$ )

$x \varepsilon^{+} F:=x \varepsilon^{1} F$

(,x ist im höchsten Grade $F:=x$ ist mindestens im Grade $1 F$ )

(Statt „die F-heit trifft auf $x$ zu“, d.h. $\lambda F(x)$, sagt Platon ,x erhält den Beinamen von der F-heit" oder , $\mathrm{X}$ wird nach der F-heit benannt“.) Aus A9 ergibt sich unmittelbar $\exists x B(x)$ - „Es gibt Begriffe“, was wir bisher nicht beweisen konnten. A10 ist das Abstraktionsprinzip in der Gestalt, in der es schon Platon kannte (,x ist (mindestens) im Grade $r$ F genau dann, wenn die F-heit (mindestens) im Grade $r$ auf $x$ zutrifft"); man kann Platon mit Fug und Recht als dessen Entdecker 
Entdecker ansehen. Aus A10 ergibt sich als Spezialfall $\forall x(x \in F$ äqu. $\lambda F(x))-, x$ ist $F$ genau dann, wenn die $F$-heit auf $x$ zutrifft“; daraus erhält man, wenn $S$ (lies „schön“) ein Beiname unserer Sprache ist, mit A9, T2 und A10:

$\lambda S \varepsilon S$ - „die Schönheit ist schön“; und man erhält $\forall x(\mathrm{~N}(\mathrm{x})$ imp. (x $\varepsilon S$ äqu. x par $\lambda S)$ ) - „Gewöhnliche Gegenstände sind genau dann schön, wenn sie an der Schönheit partizipieren“.

6.

Die Begriffe haben wir mit Platon als Standardgegenstände gedeutet. Es scheint einer gewissen Beliebigkeit zu unterliegen, welche Gegenstände als Standardgegenstände anzusehen sind. Postuliert man aber Kriterien für Standardgegenstände im Sinne von Platons Paradigma der Prädikation, so ergibt sich zwangsläufig Platons Ideenlehre:

(I) Standardgegenstände sind solche Gegenstände, die zu einer für die Beschreibung der Welt (und andere Zwecke) fruchtbaren $\mathrm{Zu}$ sammenfassung der Gegenstände in Ähnlichkeitskreise brauchbar sind; deshalb sind viele von ihnen in der Umgangssprache (unserem Instrument zur Beschreibung der Welt) schon indirekt repräsentiert, nämlich in den Adjektiven - eine Tatsache, der bei der Bestimmung der Standardgegenstände Rechnung zu tragen ist und der Platon in den Prinzipien A9 und A10 auch Rechnung trägt.

(II) Standardgegenstände dürfen nur einen Aspekt haben. Daraus ergibt sich der formhafte Charakter von Standardgegenständen. Man sieht leicht, daß materielle Gegenstände als Standardgegenstände gänzlich ungeeignet wären, denn materielle Gegenstände haben stets mehr als einen Aspekt. Welcher materielle Gegenstand könnte beispielsweise die Röte (die Rotheit) sein? - Prima vista könnte etwas wunderschön Rotes die Röte sein; aber gleichzeitig wird dieses Rote auch z.B. rund sein und eine gewisse mittlere Größe haben, und so wird nach A10 nicht nur ein rotes, riesiges Viereck nicht rot sein, sondern auch ein rosa Kreis mit der gewissen mittleren Größe rot. - (II) beinhaltet also, daß Standardgegenstände uns in der Erfahrung nicht begegnen. 
(III) Standardgegenstände müssen unveränderlich sein. Wären Standardgegenstände veränderlich, so wären sie genauso unbrauchbar wie ein nichtstarres Urmeter. Obwohl sich der Gegenstand $x$ in keiner Weise, soweit es an ihm liegt, geändert hat, erhielte man mit einem „F“ entsprechenden sich verändernden Standardgegenstand $\lambda F$ leicht, da $B \times$ zum einen Zeitpunkt $F$ ist, zum anderen nicht, wobei "F' aber ein Beiname ist, der von $x$ nach Maßgabe von $x$ allein ausgesagt wird (, $\mathrm{F}^{\mathrm{F}}$ steht also für eine intrinsische Eigenschaft von Gegenständen).

7.

Das inhaltliche Problem bei Platons Prädikationsparadigma ist, daß, wenn die Standardgegenstände, d.h. die Begriffe, die Ideen, im Einklang mit (I)-(III) bestimmt werden, es unklar wird, in welchem Sinne ein gewöhnlicher Gegenstand einem Begriff ähnlich sein soll. Dieses Problem allerdings ist nur die platonische Ausformung eines allgemeineren Problems, nämlich: In welcher Weise sind Begriffe (sei es in welcher Deutung auch immer) auf Gegenstände bezogen? Es stellt sich uns mit besonderer Schärfe, denn wir sagen nicht mit Platon „Begriffe sind ganz andere Gegenstände als andere Gegenstände“, sondern „Begriffe sind etwas ganz anderes als Gegenstände". Trivialerweise besteht die Beziehung zwischen Begriffen und Gegenständen natürlich darin, da $B$ Begriffe auf Gegenstände zutreffen; aber worin besteht die Natur dieser Beziehung? Nach Maßgabe welcher Gegebenheiten kommen wir dazu zu sagen „Begriff $f$ trifft auf Gegenstand $x$ zu“? Die Rede von Erfüllung oder Sättigung ist eine bloße Metapher; Platon aber hatte auf diese Frage eine diskutable nichtmetaphorische Antwort, ganz abgesehen davon, daß er überhaupt der Entdecker der Begriffe war. Freilich ist Platons Begriffskonzeption, die sich ausschließlich an Begriffen orientiert, die durch umgangssprachliche Adjektive (,schön“, ,gut“, ,gerecht“ etc.) repräsentiert werden, viel zu eng, und dementsprechend hat seine Prädikationstheorie, die auf diese Begriffskonzeption zugeschnitten ist, nur einen engen Anwendungsspielraum. Begriffe, die durch umgangssprachliche Substantive repräsentiert sind, und deren Beziehung zu den Gegenständen, auf die sie zutreffen, können von ihr nicht adäquat beschrieben werden. Sei M (lies „Mensch“, bzw. „Wasser") ein Beiname unserer formalen Sprache; dann gilt 
nach A9, T2 und A10 $\lambda \mathrm{M} \varepsilon \mathrm{M}$ - „das Menschsein ist Mensch“, bzw. „das Wassersein ist Wasser"; was absurd ist. (Vgl. hierzu Parmenides $130 c$, wo Platon selbst die Grenzen seiner Prädikationstheorie klargeworden sind.) Den Begriff des Menschen kann man nicht mit einem Standardgegenstand identifizieren, denn dieser Standardgegenstand müßte ein Mensch sein, aber gleichzeitig die Kriterien für Standardgegenstände erfüllen, was unmöglich ist. (Übrigens ist Platons Prädikationstheorie nicht einmal bei allen Begriffen adäquat, denen Adjektive entsprechen, sondern nur bei denen, denen sinnvoll steigerbare Adjektive zugeordnet sind; den Begriff der Schwangerschaft kann man offensichtlich ebenfalls nicht als Standardgegenstand auffassen.)

8.

Gegenüber neueren anderen Ansätzen zur Rekonstruktion der platonischen Ideenlehre und Prädikationstheorie, insbesondere dem von D.P. Ellermann („Category Theory and Concrete Universals“, Erkenntnis 28 (1988), 409-429) hat der meinige den Vorteil der größeren interpretatorischen Nähe zu Platon und der größeren Einfachheit. Nach meinem Ansatz ist auch das sogenannte Argument vom dritten Menschen definitiv als erledigt anzusehen. Ich gebe es hier für den Beinamen ,gerecht" an, nachdem wir ja die platonische Begriffslehre als ausschließlich anwendbar auf Begriffe, denen Beinamen (Adjektive) entsprechen, erkannt haben:

Die gerechten gewöhnlichen Gegenstände sind gerecht, weil sie an der Gerechtigkeit ${ }_{1}$ teilhaben; die gerechten gewöhnlichen $\mathrm{Ge}$ genstände und die Gerechtigkeit ${ }_{1}$ sind aber gerecht, weil sie an der weiteren Gerechtigkeit ${ }_{2}$ teilhaben; und wiederum: die gerechten gewöhnlichen Gegenstände, die Gerechtigkeit ${ }_{1}$ und die Gerechtigkeit $_{2}$ sind gerecht, weil sie an der Gerechtigkeit ${ }_{3}$ teilhaben; etc. etc. ad infinitum.

Doch die infinite Hierarchie von Gerechtigkeiten läßt sich leicht vermeiden. Man muß nur beachten, daß die Beziehung der Teilhabe allein zwischen gerechten gewöhnlichen Gegenständen und der Gerechtigkeit $_{1}$ besteht; die Gerechtigkeit ${ }_{1}$ aber ist nicht gerecht, weil sie an der Gerechtigkei $\hat{\imath}_{2}$ teilhat, sondern sie ist es nach A10 deshalb, weil sie ein Begriff (Standardgegenstand) und sich selbst in höchstem Grade ähnlich ist, also auf sich selbst zutrifft. Der Regreß der 
Gerechtigkeiten reißt also schon nach dem 1. Glied ab; schon eine zweite Gerechtigkeit braucht Platon nicht anzunehmen.

Dazu ist es, wie man sieht, weder nötig, die Selbsprädikation aufzugeben, noch braucht man die von G. Vlastos sogenannte Nichtidentitätsthese (siehe G. Vlastos, Platonic Studies, S. 351) zu leugnen: „NI If anything has a given character by participating in a Form, it is not identical with that Form". Ellerman hält die Leugnung der Nichtidentitätsthese für nötig (siehe „Category Theory and Concrete Universals“, S. 425), was Platon nicht gerecht wird, denn er hat die Nichtidentitätsthese mit Sicherheit vertreten (siehe das dritte und vierte Zitat im Appendix). (Noch in einem weiteren Punkte ist Ellermans Rekonstruktion interpretatorisch inadäquat: Seine konkreten Universalien können aus dem Grunde nicht ohne weiteres platonische Formen modellieren, weil nach ihm konkrete Universalien an sich selbst teilhaben [vgl. „Category Theory and Concrete Universals“, S. 413]; platonische Formen haben nicht an sich selbst teil.)

\section{9.}

Die axiomatische Theorie, die ich angegeben habe, ist in dem Sinne trivial, daß ein Widerspruchsfreiheitsbeweis für sie keinerlei Problem darstellt. Man nehme an, der Objektbereich umfasse außer den reellen Zahlen als einziges Objekt ein beliebiges Objekt b. $E(\alpha)$ ist die Extension von $\alpha$. Man setze $\mathrm{E}(\mathrm{R}(\mathrm{x}))=$ die Menge der reellen Zahlen, $E(B(x))=E(G(x))=$ die Einermenge mit $b, E(S(x, y, r))=d i e$ Menge aller Tripel $\langle b, b, i>$ wo i eine reelle Zahl: $0 \leq i \leq 1$; für alle Zahlennamen $\alpha: \mathrm{E}(\alpha) \varepsilon$ der Menge der reellen Zahlen, Standard-Zahlennamen mögen ihre Standardextension haben: $E(, 1 ")=1$ etc.; $E\left(r^{*}\right)=0.6$; für alle Beinamen $F: E(\lambda F)=b$; für alle Beinamen $F: E(x$ $\left.\varepsilon^{r} F\right)=$ die Menge aller Paare $\langle b$,i $>$, wo i eine reelle Zahl: $0.6 \leq i \leq 1$; für alle Beinamen $F$ : $E\left(x \varepsilon^{=r} F\right)=$ die Einermenge mit $<b, 1>$. Man verifiziert dann leicht die Axiome A1 - A10.

Überraschend ist angesichts dessen, $\mathrm{da} B$ es in der platonischen Prädikationstheorie möglich ist, die Existenz Gottes zu beweisen. Kein Wunder, da $B$ die platonisierende Theologie des Mittelalters in der Existenz Gottes kein Problem sah (auch Thomas von Aquin platonisiert übrigens, wenn er von Gott spricht); denn zwei Grundsätze gelten in dieser Theologie: (1) Deus est summum bonum (siehe 
z.B. Summa Theologiae, I,6,2); (2) Ens et bonum convertuntur (siehe z.B. Summa Theologiae, I,5,1), was man wie folgt umsetzen kann:

(1') Gott ist derjenige Gegenstand, der im höchsten Grade gut ist; formal: $g=\operatorname{lx}\left(G(x)\right.$ u. $\left.x \varepsilon^{+} P\right)$.

(2') Jeder Gegenstand ist genau im dem Grade gut, in dem er existent ist, und umgekehrt; formal: $\forall x\left(G(x)\right.$ imp. $\forall r\left(x \varepsilon^{=r} P\right.$ äqu. $\left.x \varepsilon^{=r} E\right)$ ).

"Gut" ist hier natürlich keine moralische Qualität, sondern kennzeichnet mit seinen Graden den Ort eines existenten Wesens in der ontologischen Stufenleiter: den Grad seiner Seinsvollkommenheit; jedes vernunftgebrauchende Wesen ist in höherem Grade gut (seinsvollkommen) als jedes nur Bewußtsein habende Wesen, jedes bewußtseinhabende Wesen ist in höherem Grade gut als jedes nur lebendige Wesen, jedes lebendige Wesen ist in höherem Grade gut als jedes nur existente Wesen.

Es läßt sich nun zeigen: $\exists ! x\left(G(x)\right.$ u. $\left.x \varepsilon^{+} P\right)-$, ,Es gibt genau einen Gegenstand, der im höchsten Grade gut ist": denn $G(\lambda P) u$. $\lambda P \varepsilon^{+} P$ (die Gutheit ist in höchstem Grade gut); nun angenommen $\mathrm{G}(\mathrm{y})$ u. y $\varepsilon^{+} \mathrm{P}$, also mit $A 10 \lambda \mathrm{P}^{+}(\mathrm{y})$, also $\lambda \mathrm{P}^{1}(\mathrm{y})$, also $\mathrm{S}(\mathrm{y}, \lambda \mathrm{P}, 1)$, also mit $A 7 y=\lambda P$. $-D a$ es genau einen Gegenstand gibt, der im höchsten Grade gut ist, folgt aber kennzeichnungslogisch: der Gegenstand, der im höchsten Grade gut ist, ist ein Gegenstand, der im höchsten Grade gut ist; folglich mit (1') Gott ist ein Gegenstand, der im höchsten Grade gut ist, also mit (2') Gott ist im höchsten Grade existent - q.e.d.

Da Gott ein Gegenstand ist, der im höchsten Grade existent ist, existiert er nicht nur, sondern ist sogar das Dasein (die Existenz) selbst; denn jeder Gegenstand, der im höchsten Grade existent ist, muß ja mit der Existenz identisch sein. Auch dies ist eines der Lehrstücke der mittelalterlichen Theologie (siehe Summa Theologieae, $1,3,4)$. 


\section{Appendix von Platonzitaten}

Wer nämlich bisher in der Liebe erzogen ist, das mancherlei Schöne in solcher Ordnung und richtig schauend, der wird, indem er nun der Vollendung der Liebeskunst entgegengeht, plötzlich ein von Natur wunderbar Schönes erblicken, nämlich jenes selbst, o Sokrates, um deswillen er alle bisherigen Anstrengungen gemacht hat, welches zuerst immer ist und weder entsteht noch vergeht, weder wächst noch schwindet, ferner auch nicht etwa nur insofern schön, insofern aber häßlich ist, noch auch jetzt schön und dann nicht, noch in Vergleich hiermit schön, damit aber häßlich, noch auch hier schön, dort aber häßlich, als ob es nur für einige schön, für andere aber häßlich wäre. Noch auch wird ihm dieses Schöne unter einer Gestalt erscheinen, wie ein Gesicht oder Hände oder sonst etwas, was der Leib an sich hat ... sondern an und für und in sich selbst ewig überall dasselbe seiend, alles andere Schöne aber an jenem auf irgendeine solche Weise Anteil habend, daß, wenn auch das andere entsteht und vergeht, jenes doch nie irgendeinen Gewinn oder Schaden davon hat, noch ihm sonst etwas begegnet. (Symposion, 211a-211b)

Wir nennen doch etwas gleich - ich meine nicht ein Holz dem andern oder einen Stein dem andern noch irgendetwas dergleichen, sondern außer diesem allen etwas anderes, das Gleiche selbst ... Scheinen sie [gleiche Hölzer] uns ebenso gleich zu sein, wie das Gleiche selbst, oder fehlt etwas daran, daß sie so sind wie das Gleiche, oder nichts? - Gar viel, sprach er, fehlt daran. ... Notwendig also kennen wir das Gleiche schon vor jener Zeit, als wir zuerst, Gleiches erblickend, bemerkten, daß alles dergleichen strebe zu sein wie das Gleiche, aber doch dahinter zurückbleibe. ... so erkannten wir auch schon, ehe wir wurden und sobald wir da waren, nicht das Gleiche nur und das Größere und Kleinere, sondern alles dieser Art insgesamt. Denn es ist uns ja jetzt nicht mehr von dem Gleichen die Rede als auch von dem Schönen selbst und dem Guten selbst und dem Rechten und Frommen und, wie ich sage, von allem, was wir bezeichnen als „dies selbst, was ist“. (Phaidon, 74a, 74d, 75a, 75c-75d) 
Mir scheint nämlich, wenn irgend etwas anderes schön ist außer jenem Schönen selbst, daß es wegen gar nichts anderem schön sei, als weil es teilhabe an jenem Schönen, und ebenso sage ich von allem.

(Phaidon, 100c)

... nachdem ihm dieses eingeräumt und zugestanden war, daß jeglicher Begriff etwas sei an sich und durch Teilnahme an ihnen die anderen Dinge den Beinamen von ihnen erhalten ... (Phaidon, 102b)

\section{Literatur}

Platon: Sämtliche Werke, 6 Bände, in der Übersetzung von F. Schleiermacher, hrsg. von E. Grassi et al., Hamburg: Rowohlt 1982.

Thomas v. Aquin: Summa Theologiae

Ellermann, D.P.: „Category Theory and Concrete Universals“, Erkenntnis 28 (1988), 409-429.

Geach, P.T.: „The Third Man Again“, Philosophical Review 65 (1956), 72-82.

Vlastos, G: Platonic Studies, Princeton, N.J.: Princeton University Press, 1981. 